Case reports

\title{
A woman with partial seizures and an unusual cerebral mass
}

Archie A Darbar MB BS, FRACP, FRCPA Infectious Diseases Physician

Luke A Coyle MB BS, FRACP, FRCPA Haematologist

Royal North Shore Hospital Sydney, NSW.

ADarbar@nsccahs. health.nsw.gov.au

MJA 2011; 195: 473- 475 doi: 10.5694/mjal0.11161

\section{Clinical record}

A frail 68-year-old woman of European ancestry presented to the emergency department with partial seizures of her left hand and mild left hemiparesis. Magnetic resonance imaging (MRI) scans of the brain revealed a non-enhancing lesion in the right precentral gyrus that was thought to represent infarction (Box, A and B).

Over the following 2 months the lesion increased in size. A brain biopsy was performed, as the patient experienced ongoing seizure activity despite anticonvulsant therapy.

The patient was married to a retired police officer and had three children. She had been raised in a country town and her father had kept cattle, pigs and sheep on their property and slaughtered meat. She had trained as a nurse and had worked in a tuberculosis ward. During early adulthood she had visited Papua New Guinea for 2 months.

The patient's background medical history was extensive. Coeliac disease was diagnosed 16 years before her current presentation. Within the past 4 years she was diagnosed with ulcerative proctitis, was twice hospitalised for pneumonia, was treated for bronchiectasis and required bone resorptive therapy for osteoporosis. She had had a hysterectomy 25 years previously.

Results of the brain biopsy were inconclusive; it showed an unusual lymphoid infiltrate and the presence of cytological atypia. There was no evidence of necrosis, malignancy or granulomas; and no organisms were noted on stains for bacteria, fungi, mycobacteria and parasites. The slides were referred for a second opinion. Dexamethasone therapy was added to help reduce the frequency of seizures.

A chest $x$-ray showed right apical pleural thickening consistent with past tuberculosis exposure, and a computed tomography (CT) scan of the abdomen showed small axillary and intra-abdominal lymph nodes; no hepatosplenomegaly or mass lesions were seen. A positron emission tomography scan showed a focus in the bowel. She was referred to a haematologist due to suspicion of lymphoma.

The patient was cachectic and weighed $38 \mathrm{~kg}$. Clinical examination showed no evidence of lymphadenopathy or hepatosplenomegaly. Results of serum biochemical analysis were normal except for a low albumin level of $30 \mathrm{~g} / \mathrm{L}$ (reference range, [RR], 37-46 g/L). She had a haemoglobin concentration of $82 \mathrm{~g} / \mathrm{L}(\mathrm{RR}, 115-160 \mathrm{~g} / \mathrm{L})$ and a normal white cell count. A blood film showed some atypical lymphocytes. Iron studies were not suggestive of iron deficiency and there was no haemolysis present. The Creactive protein level was $15 \mathrm{mg} / \mathrm{L}(\mathrm{RR},<5 \mathrm{mg} / \mathrm{L})$ and the erythrocyte sedimentation rate was $100 \mathrm{~mm} / \mathrm{h}$ (RR, $<15 \mathrm{~mm} / \mathrm{h}$ ). Paraprotein was not observed on immune electrophoresis.

An HIV test was positive on two occasions with a confirmatory western blot immunoassay. The CD4 count was $214 \times 10^{6}$ cells/L with a plasma HIV viral load of 382000 copies/mL $\left(\log _{10}\right.$ 5.58). Serological testing was negative for hepatitis $\mathrm{B}$, hepatitis $\mathrm{C}$, syphilis, Toxoplasma

\section{Magnetic resonance imaging of the brain}
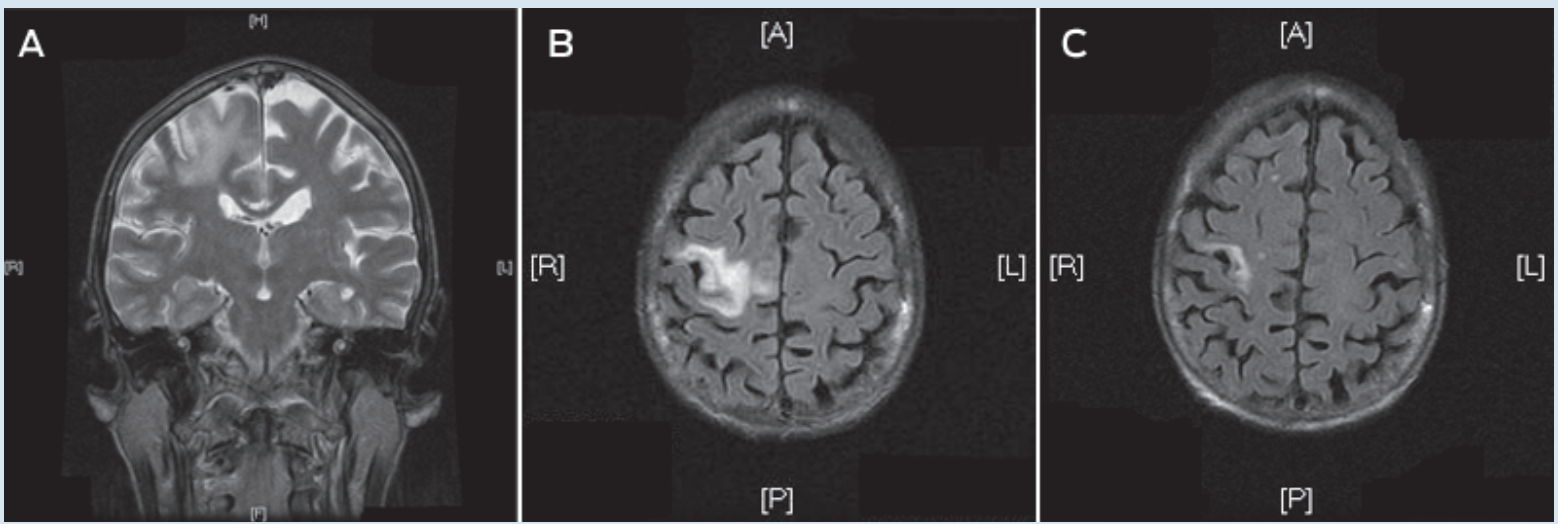

A: T2 coronal sequence at initial presentation. B: Axial fluid-attenuated inversion recovery (FLAIR) sequence at initial presentation. C: Axial FLAIR sequence after 2 months of highly active antiretroviral therapy. 
gondii infection, cryptococcal infection, hydatid disease, toxocariasis and schistosomiasis, although there was evidence of past exposure to Epstein-Barr virus and cytomegalovirus.

HIV infection was likely to have been acquired during a blood transfusion after her hysterectomy 25 years earlier. Subsequently, an Australian Red Cross Blood Service lookback confirmed that one of two units she received was from a blood donor who subsequently died of HIV-related complications. Her husband was a regular blood donor and had repeatedly screened negative for HIV infection at each donation.

Upon further review, the brain biopsies were thought to be consistent with progressive multifocal leukoencephalopathy (PML) with a florid mononuclear infiltrate, atypical astrocytes and prominent eosinophils. Immunohistochemical analysis was positive for John Cunningham virus (JCV). The patient's cerebrospinal fluid (CSF) was acellular and had normal biochemical features, but the HIV viral load was 51200 copies/mL, suggesting significant HIV replication in the brain. Polymerase chain reaction did not detect JCV DNA in the CSF.

Follow-up gastroscopy revealed lymphocytic gastritis and moderate villous atrophy of the small bowel mucosa, but no malignancy or parasitic infection. Colonoscopy revealed synchronous adenocarcinomas. After careful consideration, the patient underwent proctocolectomy with ileostomy formation. Histopathology revealed tumournode-metastasis (TNM) stage III disease with clear margins of excision and minimal node involvement. The colon had a diffuse chronic inflammatory mucosal infiltrate but this was not suggestive of ulcerative colitis, and there was no parasitic or opportunistic infection. She was assessed as not requiring adjuvant chemotherapy.

After surgery, the patient began highly active antiretroviral therapy (HAART) with a regimen comprising Trizivir (abacavir, lamivudine and zidovudine) and ritonavirboosted indinavir. She experienced symptomatic improvement, weight gain and reduced partial seizures within a few weeks. The plasma HIV viral load was undetectable within 8 weeks of commencing HAART and a repeat MRI scan at that time confirmed a significant reduction in size of the cerebral lesion (Box, C).

Unfortunately, at the same time, routine biochemistry revealed acute renal failure with an elevated serum creatinine of $295 \mu \mathrm{mol} / \mathrm{L}$ (RR, 50-90 $\mu \mathrm{mol} / \mathrm{L})$. An abdominal CT scan showed hypodense liver lesions, a large peripancreatic lesion, an enhancing lesion in the left renal collecting system, and a mass lesion encasing the left ureter. There was a significant amount of abdominal ascites. A fineneedle aspirate confirmed the presence of metastatic adenocarcinoma. Comfort measures were instituted and the patient died 4 days later.

The last case of HIV transmission from an infected blood transfusion in Australia was recognised by the Blood Service 9 years before this one (Australian Red Cross Blood Service, personal communication), and further cases from the prescreening era (before 1985) were thought unlikely to be identified due to the passage of time. This case of transfusion-acquired HIV infection was missed by routine
Blood Service tracking and the family of the deceased patient were eligible for compensation.

\section{Discussion}

Our patient presented with PML as her AIDS-defining illness with synchronous colonic neoplasms on a background of multiple medical conditions. With hindsight, these conditions were all linked to immunosuppression due to unrecognised HIV infection. It is likely that prolonged HIV replication in sanctuary sites, such as the gastrointestinal tract and brain, were responsible for her small bowel, colonic and central nervous system (CNS) disease manifestations. Impaired immune dysregulation may have contributed to the aggressiveness of the tumour behaviour.

PML is a demyelinating disease of the CNS caused by JCV, a human polyomavirus. The condition generally occurs in the setting of prolonged immunosuppression among individuals with decreased cell-mediated immunity, such as HIV infection. ${ }^{1}$ In the pre-HAART era, the prognosis for PML was very poor, with median survival times no greater than 6 months. ${ }^{2}$ With the advent of HAART, the survival time has improved to 15 months or more, but mortality rates can still be as high as 30\% to $50 \% .^{3}$ PML has also been documented among patients who receive certain immunosuppressive drugs, such as fludarabine, rituximab, corticosteroids, ${ }^{2}$ and shortly after the introduction of HAART as a form of immune-reconstitution inflammatory syndrome. ${ }^{4}$ In HIV infection, HAART is the only therapeutic option for PML, but the efficacy of these agents in controlling JCV replication in the CNS is variable and clinical response is not uniform. Antiretroviral agents with good CNS penetration have been used to optimise therapy for patients with PML. ${ }^{3}$

Previous studies have suggested the mean time to AIDS diagnosis from HIV acquisition after transfusion was 7 years, ${ }^{5}$ although a cohort of "non-progressors" who acquired a variant strain of HIV containing the nef gene deletion has been well documented. ${ }^{6}$ Our patient had delayed progression to AIDS, even in the absence of HAART, a clinical pattern that has been linked to several other genetic factors, including chemokine coreceptor type 5 (CCR5) status. ${ }^{7,8}$ CCR5 is a coreceptor required for HIV to enter $\mathrm{T}$ cells and macrophages. Homozygosity for the $\Delta 32$ gene deletion in the CCR5 gene (CCR5- $\Delta 32)$ is associated with resistance to HIV infection (although people can still be infected with T-tropic strains of the virus, which use the CXC chemokine receptor type 4 for cell entry), whereas heterozygosity confers delayed progression to disease. Our patient was confirmed as being heterozygous for CCR5432. In the United States, the frequency of the allele is $11 \%$ among white people and $1.7 \%$ among black people. ${ }^{8}$ Targeting the CCR5 receptor to interrupt HIV transmission offers new therapeutic possibilities. Maraviroc, a CCR5 inhibitor, has been introduced with success when used as part of a HAART regimen for patients with R5 (M-tropic) virus. ${ }^{9}$ Transplantation of stem cells from a donor homozygous for CCR5- $\Delta 32$ to a patient with acute myeloid leukaemia and HIV infection resulted in continued viro- 
logical suppression after transplantation in the transplant recipient and discontinuation of HAART. ${ }^{10}$

Our case highlights problems associated with delayed diagnosis of HIV infection due to the absence of perceived risk factors. In Australia, rates of HIV screening in the community, outpatient and inpatient clinical settings appear to remain suboptimal. Factors associated with decreased uptake of HIV testing include issues surrounding consent and counselling, or lack of appreciation of risk exposures by doctors and patients. A reduced threshold for performing HIV testing in these settings is required. We recommend an HIV screening test in the workup of all patients with cerebral lesions or an unusual illness pattern when the diagnosis is not easily apparent.

One approach to help identify individuals who are unaware that they have HIV infection is the "opt-out" HIV testing strategy proposed by the US Centers for Disease Control and Prevention in 2006. ${ }^{11}$ All people aged 13-64 years in health care settings would have routine HIV testing. Importantly, pretest counselling and signed consent would not be required, and the test would be performed unless the patient declined. Patients with known risks for HIV infection should be tested annually. ${ }^{11}$ This testing strategy would allow undiagnosed patients earlier access to medical care with an anticipated reduction in HIV transmission and infection related morbidity and mortality. ${ }^{12}$

The World Health Organization endorsed these recommendations in 2007, expanding the scope of uptake to the developing world. ${ }^{13}$ Drawbacks to this approach include concerns about stigmatisation and discrimination of individuals with $\mathrm{HIV}^{14}$ and increased cost implications ${ }^{15}$ for health authorities from increased test numbers, including confirmatory immunoblot assays, and a requirement for more expertise to interpret true and indeterminate results.

We encourage further debate about improved uptake of HIV testing for the Australian health setting by the appropriate governing bodies.
Acknowledgements: We thank Dr Janice Brewer, Senior Pathologist from the Department of Anatomical Pathology at Royal North Shore Hospital, and Dr Michael Buckland, Honorary Associate of Pathology at the University of Sydney based at the Department of Neuropathology, Royal Prince Alfred Hospital for providing expert histopathological assessment.

Competing interests: No relevant disclosures.

1 Demeter LM. JC, BK, and other polyomaviruses; progressive multifocal leukoencephalopathy. In: Mandell GL, Bennett JE, Dolin R, editors. Principles and practice of infectious diseases. 7th ed. Philadelphia: Churchill Livingstone, 2009.

2 Weber T. Progressive multifocal leukoencephalopathy. Neurol Clin 2008; 26 : 833-854.

3 Falcó V, Olmo M, del Saz SV, et al. Influence of HAART on clinical course of HIVl-infected patients with progressive multifocal leukoencephalopathy: results of an observational multicentre study. J Acquir Immune Defic Syndr 2008; 49: 26-31.

4 Safdar A, Rubocki RJ, Horvath JA, et al. Fatal immune restoration disease in human immunodeficiency virus type l-infected patients with progressive multifocal leukoencephalopathy: impact of antiretroviral therapy-associated immune reconstitution. Clin Infect Dis 2002; 35: 1250-1257.

5 Kopec-Schrader E, Tindall B, Learmont J, et al. Development of AIDS in people with transfusion-acquired HIV infection. AIDS 1993; 7: 1009-1013.

6 Learmont JC, Geczy AF, Mills J, et al. Immunologic and virologic status after 14 to 18 years of infection with an attenuated strain of HIV-1. A report from the Sydney Blood Bank Cohort. N Engl J Med 1999; 340: 1715-1722.

7 Hogan CM, Hammer SM. Host determinants in HIV infection and disease. Part 1: cellular and humoral immune responses. Ann Intern Med 2001; 134: 761-776.

8 Hogan CM, Hammer SM. Host determinants in HIV infection and disease. part 2: genetic factors and implications for antiretroviral therapeutics. Ann Intern Med 2001; 134: 978-996.

9 Gulick RM, Lalezari J, Goodrich J, et al. Maraviroc for previously treated patients with R5 HIV-1 infection. N Engl J Med 2008; 359: 1429-1441.

10 Hütter G, Nowak D, Mossner M, et al. Long-term control of HIV by CCR5 Delta32/Delta32 stem-cell transplantation. N Engl J Med 2009; 360: 692-698.

11 Branson BM, Handsfield HH, Lampe MA, et al. Revised recommendations for HIV testing of adults, adolescents and pregnant women in health care settings. MMWR Recomm Rep 2006; 55 (RR-14): 1-17.

12 Bartlett JG, Branson BM, Fenton K, et al. Opt-out testing for human immunodeficiency virus in the United States. Progress and challenges. JAMA 2008; 300: 945-951.

13 Bassett IV, Walensky RP. Integrating HIV screening into routine health care in resource limited settings. Clin Infect Dis 2010; 50 Suppl 3: S77-S84.

14 Dodds C, Keogh P, Chime O, et al. Outsider status: stigma and discrimination experienced by gay men and African people with HIV. London: Sigma Research, 2004. http://www.sigmaresearch.org.uk/files/report2004f.pdf (accessed Nov 2010).

15 Holtgrave DR. Costs and consequences of the US Centers for Disease Control and Prevention's recommendations for opt-out HIV testing. PLoS Med 2007; 4: e194. 